\title{
Kidney Injury Molecule-1 is Elevated in Nephropathy and Mediates Macrophage Activation via the Mapk Signalling Pathway
}

\author{
Lei Tian Xinghua Shao Yuanyuan Xie Qin Wang Xiajing Che Minfang Zhang \\ Weijia Xu Yao Xu Zhaohui Ni Shan Mou
}

Department of Nephrology, Molecular Cell Lab for Kidney Disease, Ren Ji Hospital, School of Medicine, Shanghai Jiaotong University, Shanghai, China

\section{Key Words}

Kidney injury molecule-1 1 Macrophage MAPK signaling pathway • Acute kidney injury • Chronic kidney disease

\begin{abstract}
Background/Aims: Kidney injury molecule-1 (KIM-1) is highly expressed in renal tubular cells after injury and is usually regarded as an early biomarker of acute kidney injury(AKI). The aim of this study was to determine the role of KIM-1 in the development of renal tubular injury. Methods: Clinical samples, three different animal models and in vitro experiments were utilized to determine the possible mechanism underlying the involvement of KIM-1 in kidney injury. Results: Both plasma and urinary KIM-1 expression levels were significantly higher in AKI and chronic kidney disease (CKD) patients than in healthy volunteers, and urinary KIM-1 expression was significantly higher in CKD patients than in AKI patients. According to the results of our research involving three different mouse models, KIM-1 expression was significantly increased during the early stage of kidney injury and was persistently elevated in renal fibrosis. Our immunofluorescence staining results indicated that KIM-1-positive tubules were surrounded by macrophage infiltrates in regions of kidney injury. Moreover, our transwell, western blotting and real-time PCR data showed that macrophage migration and phenotype transitions were mediated by KIM-1 through the mitogen-activated protein kinase (MAPK) pathway. MAPK pathway inhibition could significantly reverse the effects of KIM-1 with respect to these macrophage phenotype changes and migration. Conclusions: KIM-1 expression was markedly elevated in both acute and chronic kidney injury and may play a pivotal role in macrophage activation via the MAPK pathway in kidney disease.
\end{abstract}




\section{Cellular Physiology Cell Physiol Biochem 2017;41:769-783

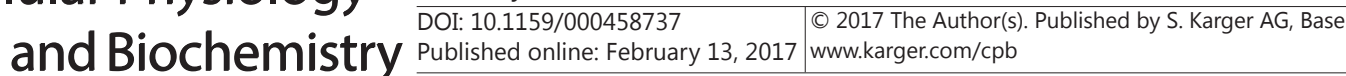 \\ Tian et al.: KIM-1 Regulates Macrophage in Nephropathy}

\section{Introduction}

Kidney disease is a worldwide public health problem affecting millions of people from all racial and ethnic groups [1]. Loss of renal function during kidney disease may be rapid and reversible, as in the case in acute kidney injury (AKI). However, in chronic kidney disease (CKD), renal function declines slowly, and no prominent symptoms develop during the early stages of the disease. Both AKI and CKD may progress to end-stage renal disease (ESRD), which requires renal replacement therapy. Despite the existence of various interventions that can delay kidney disease progression, the number of patients who reach ESRD has remained high in recent years [2]. One of the major reasons for this frustrating finding is our incomplete understanding of the mechanisms underlying the processes of regeneration and healing from kidney disease.

Tubulointerstitial injury is a common kidney disease progression pathway in which renal tubular cells play a crucial role [3]. Notably, kidney tubules possess a remarkable capacity to self-repair and regenerate following injury. Kidney injury molecule-1 (KIM-1), a type-1 transmembrane protein, is an emerging biomarker whose expression and release are induced in renal tubular cells after injury $[4,5]$. Previous reports involving rat models have shown that KIM-1 is an outstanding indicator of kidney injury and is a better predictor of kidney injury than serum creatinine [4]. Our previous studies also reported that urinary KIM-1 was an efficient novel urinary biomarker for the diagnosis of AKI [6-8]. However, few studies have focused on the function and mechanism underlying the function of KIM-1 in CKD. Thus, whether KIM-1 is "friend" or "foe" in kidney injury remains a controversial subject.

Macrophage accumulation within the kidney occurs in most types of primary and secondary human glomerulonephritis [9]. The density of macrophage accumulation correlates with the degree of renal dysfunction and is predictive of disease progression, fibrosis or tubular atrophy. The results of studies in both immune and non-immune-initiated models of kidney disease have also indicated that activated macrophage infiltration within the kidney was critically involved in the initiation and orchestration of the acute inflammatory response during kidney injury [10]. Targeting macrophages and their functions will lead to improved outcomes in many kidney diseases.

Moreover, in a clinical study of biopsy specimens from 102 cases of various renal diseases, van Timmeren et al. [11] found that KIM-1 expression was significantly induced and was localized to the apical side of dilated tubules in fibrotic areas in all renal diseases, except for minimal change disease, and that KIM-1 expression was significantly associated with macrophage infiltration. In addition, double-labelling immunohistochemistry revealed that KIM-1 was primarily present in regions with interstitial macrophages. The relationship between KIM-1 expression and macrophages is still unknown. The Bonventre group studies about KIM-1 have hypothesized that KIM-1 may play a key role in the crosstalk between injured tubular cells and macrophages $[12,13]$. Accordingly, in this study, we used three animal models, as well as in vitro experiments and clinical samples, to determine the possible role of KIM-1 in kidney injury. We used macrophage cultures to determine the potential effects of KIM-1 on macrophage migration and activation. In addition, we tested the hypothesis that patients with kidney injury have higher blood and urinary KIM-1 concentrations than healthy people and that KIM-1 regulates macrophage migration and activation via the mitogen-activated protein kinase (MAPK) signalling pathway in kidney disease.

\section{Materials and Methods}

Selection of Patient Study Groups

Single spot urine samples and corresponding plasma samples were obtained from healthy individuals, patients with AKI, and patients with CKD. Healthy volunteers from the Health Examination Center of Renji 


\section{Cellular Physiology Cell Physiol Biochem 2017;41:769-783

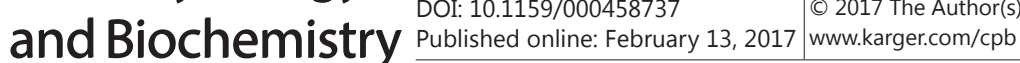 \\ Tian et al.: KIM-1 Regulates Macrophage in Nephropathy}

Hospital, Shanghai Jiao Tong University, who were free of chronic inflammatory diseases, chronic infectious diseases, and metabolic disease were enrolled in the study. The study was approved by the Ethics Committee of Renji Hospital. All participating subjects gave written consent to participate in the study, according to the Declaration of Helsinki. Patients with AKI from the Department of Urology and Nephrology, Renji Hospital, Shanghai Jiao Tong University, were also included in the study. AKI was defined as an increase in plasma creatinine of $\geq 50 \%$ compared with baseline within 7 days or an increase in plasma creatinine of $\geq 0.3 \mathrm{mg} / \mathrm{dl}$ compared with baseline within 2 days, according to the KDIGO criteria. CKD was defined as kidney damage persisting for 3 months or longer or a glomerular filtration rate lower than $60 \mathrm{~mL} / \mathrm{min}$ per $1.73 \mathrm{~m}^{2}$ for 3 months or longer. CKD urine and plasma samples were collected from outpatients attending a general nephrology clinic at Ren Ji Hospital, Shanghai Jiao Tong University.

\section{Cisplatin-induced AKI model}

Male C57/BL mice aged 8-10 weeks were injected intraperitoneally once with $15 \mathrm{mg} / \mathrm{kg}$ cisplatin (Sigma, USA) or saline alone. Plasma and tissue samples were collected and analysed on days 3, 7 and 14 after cisplatin treatment.

Aristolochic acid-induced renal fibrosis after AKI model

Male C57/BL mice aged 8-10 weeks were injected intraperitoneally once with $10 \mathrm{mg} / \mathrm{kg}$ aristolochic acids (AA) (Sigma, USA) or saline alone. Plasma and tissue samples were collected and analysed on days 3 , 7 , and 14 after treatment.

Unilateral ureteral obstruction for renal fibrosis model

Unilateral ureteral obstruction (UUO) was induced in male C57/BL mice aged 8-10 weeks, as described previously [14]. The mice were anesthetized, and the ureter of the left kidney was ligated with 6-0 silk at two points proximal to the kidney. In sham animals, flank incisions were made, and the kidney was exposed, but the ureter was not tied. The mice were euthanized on days 3, 7, 14, and 21 after surgery, and plasma and tissue samples were collected and analysed.

Histologic Evaluation of mouse kidneys from different models

Tissue samples from three animal models were fixed in 10\% neutral buffered formalin or snap-frozen. For histologic assessment, we prepared $2 \mathrm{~mm}$ paraffin sections and stained them with haematoxylin and eosin (HE) and Masson trichrome. Tubular damage in these HE-stained kidney sections was examined under a light microscope and scored based on the presence of morphologic changes signifying tubular cell necrosis (2), brush border loss (1), vacuolization (1), tubule dilation (1), cast formation (2), and inflammatory cell infiltration (1). Fibrosis in the Masson trichrome-stained kidney sections was scored according to the percentage of interstitial fibrosis as follows: no fibrosis (0), mild: less than $25 \%$ (1), moderate: less than $50 \%$ (2), severe: less than $75 \%$ (3), and very severe: more than $75 \%$ (4).

\section{Immunofluorescence staining of human kidney biopsy samples and mouse kidneys}

Two formalin-fixed kidney biopsied tissue samples from patients with AKI or CKD were costained with a KIM-1 antibody (1:100; Product number: SAB3500252 for human, Sigma, USA) and the macrophage marker CD68 (1:100; Product number: ab955 for mouse, Abcam, Cambridge, UK), respectively, whereas normal kidney tissue samples from the site opposite a tumour in one nephrectomy patient were used as controls. Mouse kidney cryosections were fixed in 4\% paraformaldehyde for $15 \mathrm{~min}$ at room temperature, followed by permeabilization with $0.4 \%$ TritonX-100 in phosphate-buffered saline for $10 \mathrm{~min}$. After being blocked with $5 \%$ bovine serum albumin in phosphate-buffered saline for $30 \mathrm{~min}$, the samples were incubated with primary antibodies against KIM-1 (1:100; Product number: BAF 1817 for mouse, R\&D Systems Europe, Abingdon, UK) and F4/80 (1:100; Product number: ab186073, Abcam, Cambridge, UK). Epifluorescence microscopy was performed with a Nikon microscope (Nikon, Melville, NY, USA).

\section{HK-2 Cell Culture and Treatments}

Human kidney tubular epithelial (HK-2) cells were purchased from American Type Culture Collection (CRL-1571; Manassas, VA) and cultured in Gibco DMEM F/12 (Life Technologies, Grand Island, NY) with $10 \%$ Gibco foetal bovine serum (Life Technologies) in an atmosphere of $5 \% \mathrm{CO} 2$ at $37^{\circ} \mathrm{C}$. The HK2 cells 


\section{Cellular Physiology Cell Physiol Biochem 2017;41:769-783

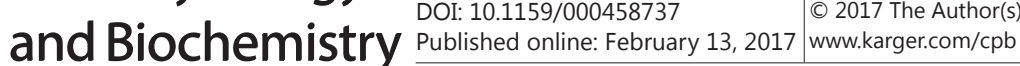

Tian et al.: KIM-1 Regulates Macrophage in Nephropathy

were starved for 24 hours by incubation with DMEM F/12 containing 0.5\% foetal bovine serum and were exposed to various experimental conditions. The cells were divided into the following groups: 1) a normal control group incubated in DMEM F/12 containing vehicle, 2) a cisplatin group stimulated with cisplatin (25 $\mu \mathrm{M}$; Sigma, USA), 3) an aristolochic acid group stimulated with aristolochic acids ( $5 \mu \mathrm{g} / \mathrm{ml}$; Sigma, USA) and 4) a transforming growth factor- $\beta 1$ (TGF- $\beta 1$ ) group stimulated with recombinant TGF- $\beta 1$ ( $10 \mathrm{ng} / \mathrm{ml}$; R\&D Systems Europe, Abingdon, UK) for 48 hours.

\section{Raw264.7 cell culture and treatment}

The RAW 264.7 cell line, a murine macrophage-like cell line, was purchased from the Cell Bank of the Chinese Academy of Sciences (Shanghai, China) and cultured in Gibco DMEM (Life Technologies, Grand Island, NY) with $10 \%$ Gibco foetal bovine serum (Life Technologies) in an atmosphere of $5 \% \mathrm{CO} 2$ at $37^{\circ} \mathrm{C}$. The Raw264.7 cells were incubated with KIM-1 (200 ng/ml, R\&D Systems Europe, Abingdon, UK) for 12 hours with or without pretreatment with specific MAPK inhibitors (U0126, SB203580, or SP600125; Sigma, USA).

Enzyme linked immunosorbent assays and other tests

KIM-1, tumour necrosis factor- $\alpha$ (TNF- $\alpha$ ), interleukin-6 (IL-6), interleukin-4 (IL-4), and interleukin-10 (IL-10) expression levels in urine, blood, and the HK-2 cell supernatants were measured by enzyme linked immunosorbent assay (ELISA) using commercially available kits, as instructed by the manufacturers (R\&D, Minneapolis, MN, USA). Moreover, changes in interferon- $\gamma$ (IFN- $\gamma$ ), inducible nitric oxide synthase (iNOS), arginase 1 (Arg-1) and mannose receptor (MR) mRNA expression levels in Raw264.7 cells and mouse kidney cortex were detected by real-time polymerase chain reaction (PCR) analysis and ELISA. Serum and urine creatinine concentrations were determined enzymatically. The glomerular filtration rate (GFR) was estimated using the simplified modified diet in renal disease (MDRD) formula, eGFR=186×(sCr/88.4) $-1.154 \times$ age $-0.203 \times(0.742$, female $)[15]$.

\section{Western Blot Analysis}

SDS-PAGE and immunoblot analyses were carried out according to standard protocols and visualized using enhanced chemiluminescence immunoblot detection kits (Amersham Pharmacia Biotech UK, Little Chalfont, Buckinghamshire, UK). Polyvinylidene fluoride membranes were incubated at $4^{\circ} \mathrm{C}$ overnight with either rabbit polyclonal or monoclonal primary antibodies targeted to one of the following proteins (with their respective dilutions): KIM-1 (1:1000; Product number: SAB3500252, Sigma, USA), a-SMA (1:1000; Product number: ab5694, Abcam, Cambridge, UK), and E-cadherin (1:500; Product number: ab76319, Abcam, Cambridge, UK), as well as the following proteins from Cell Signaling Technology (Danvers, MA): GAPDH (1:1000; Product number: 2118), phospho-p38 MAPK (1:1000; Product number: 4511), p38 MAPK (1:1000; Product number: 41666), c-Jun N-terminal kinase (JNK) MAPK (1:2500; Product number: 9252), phospho-JNK MAPK (1:2500; Product number: 9255), extracellular signal-regulated kinase 1/2 (ERK1/2) MAPK (1:1000; Product number: 4694), and phospho-ERK1/2 MAPK (1:1000; Product number: 4370).

Western blotting analysis of the urine specimens was performed as previously described [16].

\section{Cell Migration Assay}

Macrophage migration assay was conducted using a 24-well transwell unit with polycarbonate filters with a diameter of $6.5 \mathrm{~mm}$ and a pore size of $8.0 \mu \mathrm{m}$ (Corning Costar, Cambridge, MA, USA), as previously reported, with some modifications. The cells $(2 \times 105$ cells $)$ were loaded into the top chamber and were pretreated with or without specific MAPK inhibitors (U0126, SB203580, or SP600125). After KIM-1 stimulation, the chambers were incubated for 12 hours. Cells that had migrated to the lower surfaces of the filter were fixed with $20 \%$ methanol for $30 \mathrm{~min}$ and stained with $0.2 \%$ crystal violet for $30 \mathrm{~min}$. Unmigrated macrophages on the surface of the upper membrane were removed using a cotton swab. The macrophages that had migrated were quantified by counting 5 random spots in each independent sample.

\section{RNA Preparation and Real-time Quantitative RT-PCR}

Total RNA was isolated from kidney cortex of mice and raw264.7 cell using Trizol reagent (Invitrogen/ Life Technologies) and was reverse-transcribed to cDNA using a Revert Aid First Strand cDNA Synthesis Kit (Thermo Scientific, Pittsburgh, PA). The PCR primer sequences were performed as shown in Table 1. 
Table 1. Primers used for RT-PCR. Abbreviation: IFN $-\gamma$, interferon- $\gamma$; iNOS, inducible nitric oxide synthase; Arg-1, arginase 1;MR, mannose receptor

\begin{tabular}{ll}
\hline Gene name & Primer sequences \\
\hline IFN- $\gamma$ & Forward: CGGCACAGTCATTGAAAGCC \\
& Reverse: TGTCACCATCCTTTTGCCAGT \\
iNOS & Forward: GCCACCAACAATGGCAACAT \\
& Reverse: CGATGCACAACTGGGTGAAC \\
Arg-1 & Forward: ACATTGGCTTGCGAGACGTA \\
& Reverse: ATCGGCCTTTTCTTCCTTCCC \\
MR & Forward: TTTGCCTTTCCCAGTCTCCC \\
& Reverse: CCTCGCGTCCAATAGCTGAA \\
GAPDH & Forward: GCCTTCCGTGTTCCTACC \\
& Reverse: AGAGTGGGAGTTGCTGTTG \\
\hline
\end{tabular}

Table 2. Characteristics of participants in cross-sectional study. Values for continuous variables given as mean \pm SEM or mean $(95 \%$ CI). AKI, acute kidney injury . CKD , chronic kidney disease. KIM-1, kidney injury molecule-1. *Baseline eGFR was calculated using the Modification of Diet in Renal Disease equation. ${ }^{\text {a }}$ $\mathrm{P}<0.01$, versus healthy volunteers. ${ }^{\mathrm{b}} \mathrm{P}<0.01$, versus $\mathrm{AKI}$ patients

\begin{tabular}{|c|c|c|c|}
\hline Characteristic & $\begin{array}{l}\text { Healthy } \\
\text { Volunteers } \\
(\mathrm{n}=30)\end{array}$ & $\begin{array}{l}\text { AKI Patients } \\
(\mathrm{n}=30)\end{array}$ & $\begin{array}{l}\text { CKD Patients } \\
(n=30)\end{array}$ \\
\hline Age & $48 \pm 11.86$ & $54.5(27$ to 65$)$ & $58(30$ to 60$)$ \\
\hline Sex(Female/Male) & $17 / 13$ & $13 / 17$ & $11 / 19$ \\
\hline Baseline plasma creatinine $(\mu \mathrm{mol} / \mathrm{L})$ & $\begin{array}{l}58.7(53.50 \text { to } \\
78.1)\end{array}$ & $150.1(114.3 \text { to } 220)^{\mathrm{a}}$ & $374.2(268 \text { to } 499.9)^{\text {ah }}$ \\
\hline Baseline eGFR $\left(\mathrm{ml} / \mathrm{min} \text { per } 1.73 \mathrm{~m}^{2}\right)^{*}$ & $103.23 \pm 12.5$ & $41.63 \pm 19.85^{a}$ & $14(9 \text { to } 22)^{\mathrm{ab}}$ \\
\hline Plasma KIM-1 (ng/L) & $\begin{array}{l}90(83.43 \text { to } \\
104.18)\end{array}$ & $\begin{array}{l}675.97(580.56 \text { to } \\
750.63)^{\mathrm{a}}\end{array}$ & $\begin{array}{l}692.21(643.27 \text { to } \\
817.84)^{\mathrm{a}}\end{array}$ \\
\hline $\begin{array}{l}\text { Urinary KIM-1 (ng/mg urinary } \\
\text { creatinine) }\end{array}$ & $0.13(0.09$ to 0.28$)$ & $1.23(0.57 \text { to } 1.72)^{\mathrm{a}}$ & $5.15(1.28 \text { to } 8.68)^{\mathrm{ab}}$ \\
\hline
\end{tabular}

Statistical analysis

Data analysis was carried out using SPSS software (version 18: SPSS, Chicago, IL, USA). Normally distributed variables were expressed as the mean $\pm \mathrm{SD}$, and non-parametric variables were expressed as medians and ranges. The cell culture experiments were performed at least in triplicate.

\section{Results}

Increased plasma and urinary KIM-1 expression was noted in AKI and CKD patients

Thirty healthy volunteers, 30 AKI patients, and 30 CKD patients were recruited for the cross-sectional clinical study. AKI samples were chosen as close in time as possible to peak serum creatinine values. The demographic characteristics and clinical descriptions of the entire study population, as well as its serum creatinine level, eGFR, and urinary and plasma KIM-1 levels, are shown in Table 2. The results showed that both plasma and urinary KIM-1 expression levels were significantly higher in AKI and CKD patients than in healthy volunteers $(\mathrm{P}<0.01$ ) (Fig. $1 \mathrm{~A})$. Furthermore, urinary KIM-1 levels were markedly higher in CKD patients than in AKI patients $(\mathrm{P}<0.01)$ (Fig. 1,B). The scatterplot results demonstrated the existence of a negative correlation between plasma KIM-1 levels and the estimated glomerular filtration rate (eGFR) in all participants ( $\mathrm{r}=-0.725, \mathrm{P}<0.01)$ (Fig. 1,C), as well as between urinary KIM-1 levels and the eGFR ( $\mathrm{r}=-0.333$, $\mathrm{P}<0.01$ ) (Fig. 1,D). However, the eGFR was not correlated with plasma KIM-1 or urinary KIM-1 levels in AKI and CKD patients after subset analysis (Fig. 2). The western blotting results for protein concentrations in urine also indicated that urinary KIM-1 was overexpressed in both AKI and CKD patients (Fig. 1,E). These results indicated that both plasma and urinary KIM-1 levels were increased in AKI and CKD patients and may be associated with the degree of renal damage. 
A

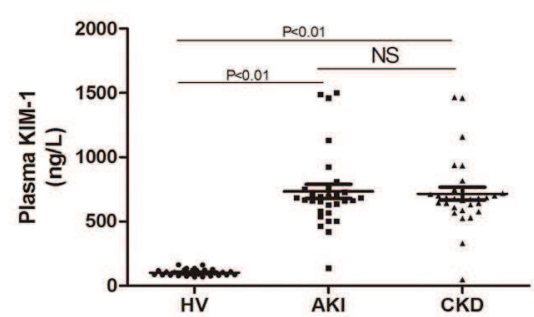

C

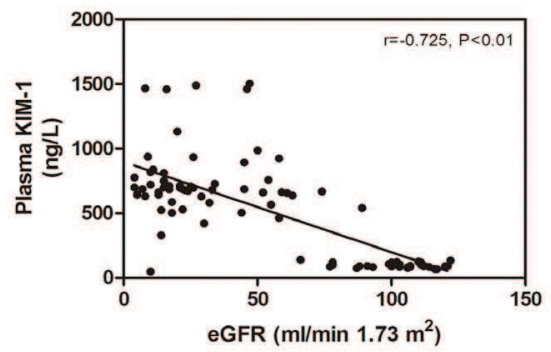

B

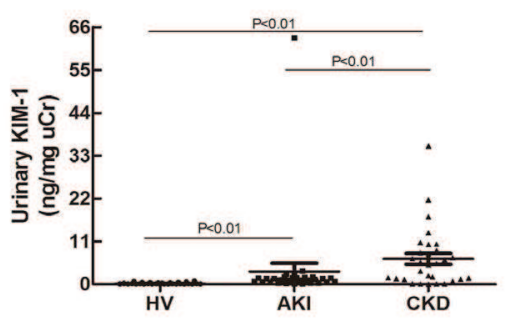

D

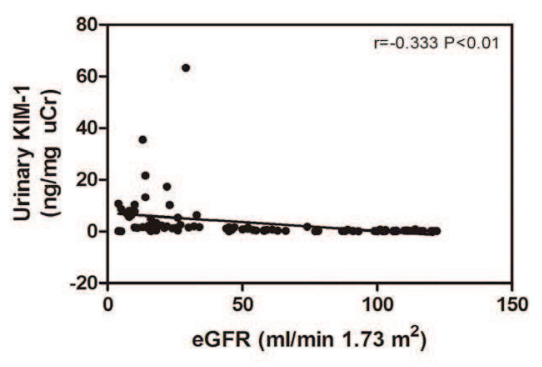

E

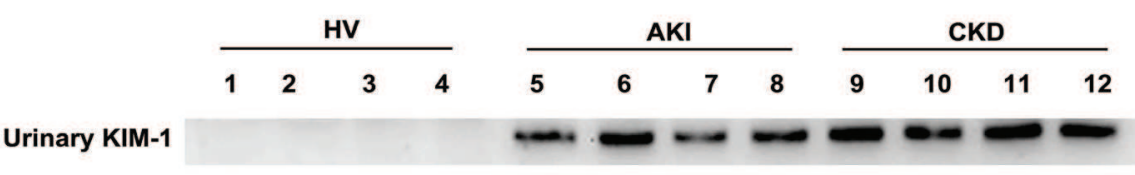

Fig. 1. Increased plasma and urinary KIM-1 expression was noted in AKI and CKD patients. Plasma and urine samples were collected from healthy volunteers (HV), acute kidney injury (AKI) patients and chronic kidney injury (CKD) patients. Dot plots indicating plasma KIM-1 (A) and urinary KIM-1 expression normalized to urinary creatinine (B) for each patient. (C) Scatter plot demonstrating a negative correlation between plasma KIM-1 expression and the eGFR in all participants, including healthy volunteers $(n=30), A K I$ patients $(n=30)$ and CKD patients $(n=30)$. $(r=-0.725 ; P<0.01)$. (D) Scatterplot demonstrating a correlation between urinary KIM-1 expression and the eGFR ( $r=-0.333$; $P<0.01)$. (E) Western blotting depicting urinary KIM-1 bands in healthy volunteers (lanes 1, 2, 3, and 4), AKI patients (lanes 5, 6, 7, and 8), and CKD patients (lanes 9, 10, 11, and 12).

Plasma KIM-1 expression was associated with kidney injury and renal function

To clarify the expression patterns of KIM-1 in renal damage, we established three kidney injury models. Renal tubule lesions were visualized by haematoxylin and eosin (HE) staining. In the cisplatin-induced AKI mouse model, extensive renal tubular injury characterized by tubule dilation, cast formation, tubular cell necrosis, and interstitial inflammatory cell infiltration was observed on day 3 after cisplatin treatment but recovered after day 14 (Fig. 3,A). Plasma creatinine levels were significantly increased after day 3 but returned to normal after day 14. Plasma KIM-1 levels were significantly elevated after day 3 of cisplatin treatment but returned to normal after day 14 (Fig. 3,D). In the aristolochic acidinduced renal fibrosis after AKI mouse model, severe kidney lesions exhibiting hyaline casts, inflammatory cell infiltration, and tubular cell necrosis were observed on day 3 after AA administration, and tubular atrophy with inflammatory cell infiltration was observed after day 14 (Fig. 3,B). Plasma creatinine levels continued to rise after day 3, while plasma KIM-1 levels rose steadily (Fig. 3,E). In the UUO-induced renal fibrosis model, tubular dilatation and inflammatory cell infiltration were present after day 3 , and tubular atrophy was prominent after day 14 (Fig. 3,C). The trends for plasma creatinine levels were similar to those for plasma KIM-1 levels (Fig. 3,F). 


\section{Cellular Physiology \\ Cell Physiol Biochem 2017;41:769-783 and Biochemistry

Fig. 2. The correlation of plasma/urinary KIM-1 and eGFR in subgroup analysis. The correlation of plasma/ urinary KIM-1 and eGFR was illustrated by scatter plot.(AB) eGFR was not correlated with plasma KIM-1 ( $\mathrm{r}=-0.004$; $\mathrm{P}=0.985)$ or urinary KIM-1 $(\mathrm{r}=0.120 ; \mathrm{P}=0.526$ )levels in healthy volunteers; (C-D) eGFR was not correlated with plasma KIM-1 $\quad(\mathrm{r}=-0.203$; $\mathrm{P}=0.282)$ or urinary KIM-1 ( $\mathrm{r}=-0.155 ; \mathrm{P}=0.413$ )levels in AKI group; (E-F) eGFR was not correlated with plasma KIM-1 ( $\mathrm{r}=0.144 ; \quad \mathrm{P}=0.449)$ or urinary KIM-1 ( $\mathrm{r}=0.174$; $\mathrm{P}=0.357$ )levels in CKD group.
A

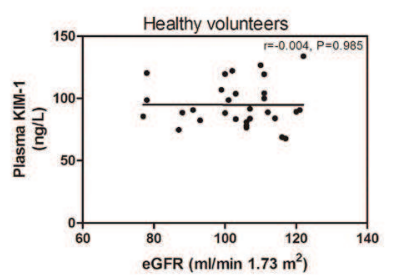

C

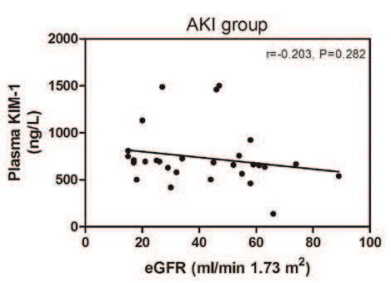

E

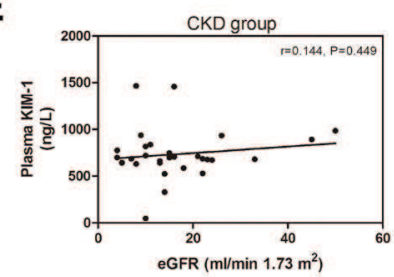

B

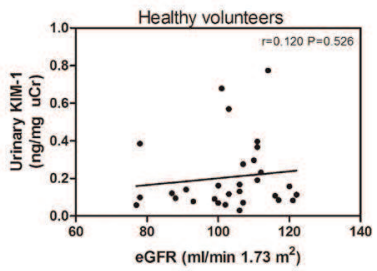

D

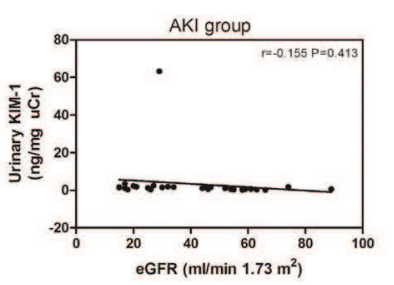

$\mathbf{F}$

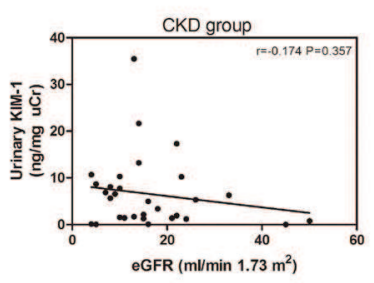

Sustained KIM-1 expression was observed in renal fibrosis

Renal interstitial fibrosis was evaluated in different mouse models by Masson trichrome staining. Renal fibrosis was not observed in the kidneys of cisplatin-challenged mice (Fig. 4,A). At day 7 after AA administration, renal interstitial fibrosis was detected. This fibrosis was aggravated after day 14 (Fig. 4,B). In the UUO-induced renal fibrosis mouse model, interstitial fibrosis and tubular atrophy worsened progressively with the passage of time (Fig. 4,C).

In addition, we evaluated the relationship between KIM-1 expression and renal fibrosis by western blotting. In the cisplatin-induced AKI mouse model, high KIM-1 expression was detected in kidney tissues at day 3 after cisplatin administration but was not detected at day 14 after administration, and no $\alpha$-smooth muscle actin ( $\alpha$-SMA) expression was detected in kidney tissues after cisplatin administration (Fig. 4,D). At day 3 after AA treatment, KIM-1 expression in kidney tissues was significantly increased, and this sustained KIM-1 expression was accompanied by $\alpha$-SMA expression (Fig. 4,E). In the UUO-induced mice model, KIM-1 and $\alpha$-SMA expression in obstructed kidneys gradually increased (Fig. 4,F). Western blot showed the changes in KIM-1 expression that occurred in the kidney tissues were different from those occurred in the plasma samples.

In vitro, the ELISA results showed that KIM-1 expression was increased in the supernatant of HK-2 cells after cisplatin $(25 \mu \mathrm{mol} / \mathrm{L})$, AA $(5 \mu \mathrm{g} / \mathrm{ml})$, or TGF- $\beta 1(10 \mathrm{ng} / \mathrm{ml})$ treatment (Fig. 5,A-C). Western blotting demonstrated the presence of KIM-1 expression in renal tubular interstitial fibrosis (Fig. 5,D-F).

KIM-1-positive staining tubules were surrounded by macrophages in renal damage areas

In the three mouse models, interstitial inflammatory cell infiltration occurred in the damaged mouse kidneys. We also observed macrophage expression in the damaged kidneys by immunofluorescence. We did not detect macrophage infiltration in the control groups. On day 3 after kidney injury in the three mouse models, extensive tubular KIM-1 expression with macrophage (F4/80 positive) infiltration was observed. KIM-1-positive tubules with macrophage infiltration were still present on day 14 after AA and UUO treatment. However, KIM-1 expression was clearly reduced on day 14 after cisplatin treatment (Fig. 6,A). 


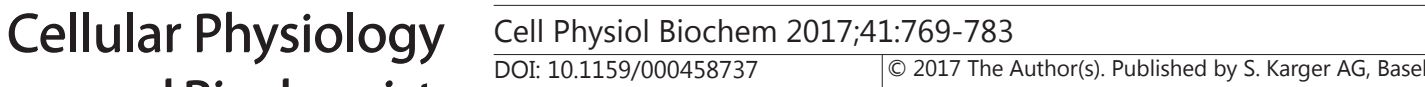

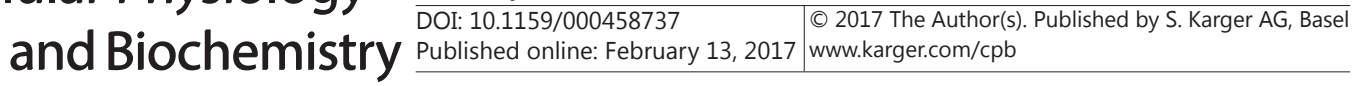

A
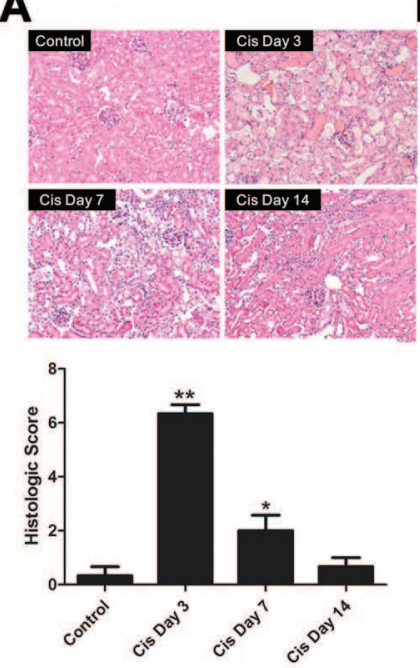

D
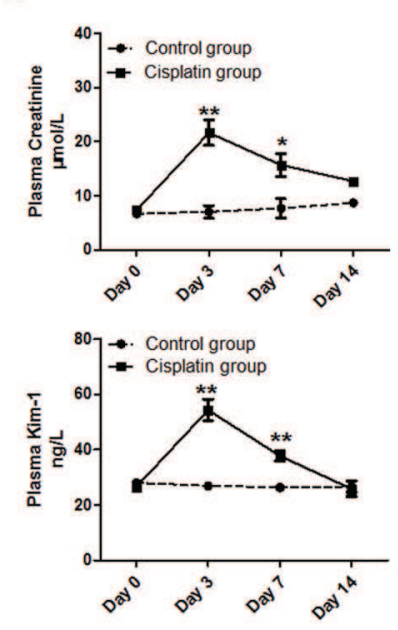

B
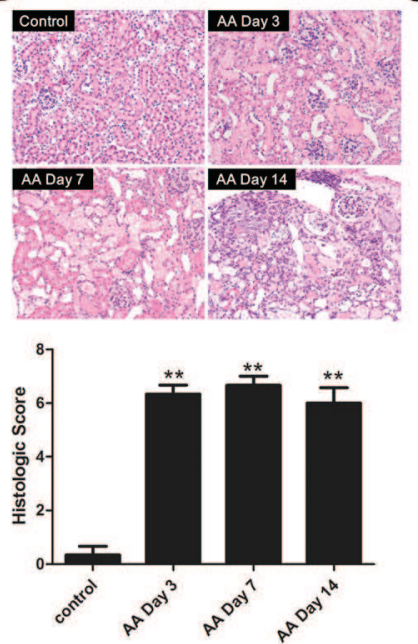

E
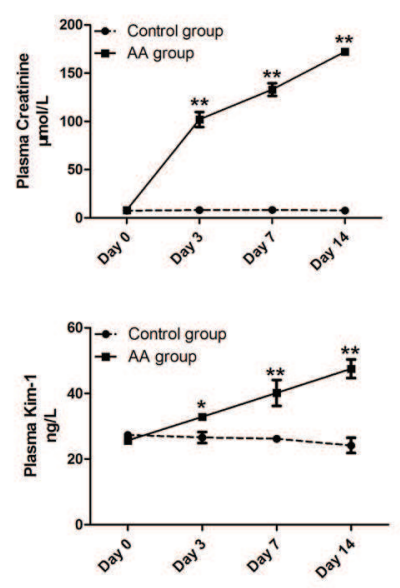

C
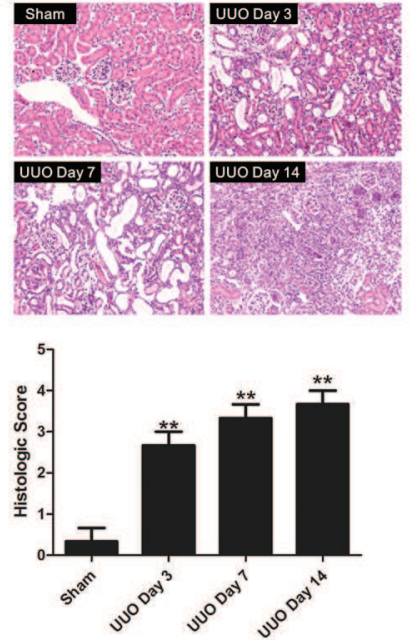

$\mathbf{F}$
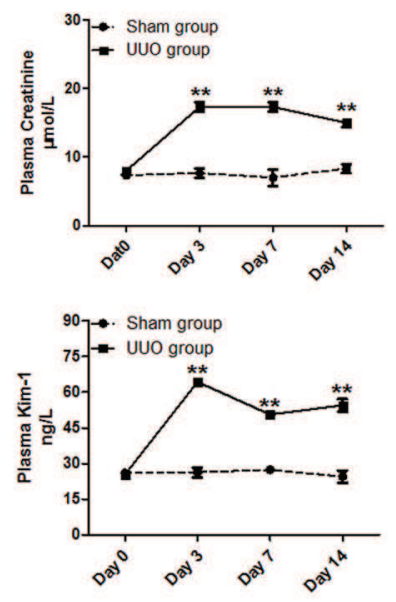

Fig. 3. Plasma KIM-1 was associated with kidney injury and renal function. (A-C)Haematoxylin and eosin (HE) staining of kidney sections and quantification of histologic scoring in vivo. (A) Male C57/BL mice were administered one dose of cisplatin (Cis) (15 mg/kg). (B) Male C57/BL mice were administered one dose of aristolochic acid (AA) (10 mg/kg). (C) Male C57/BL mice were subjected to UUO by obstructing the ureter. The kidney sections were stained with $\mathrm{HE}$ and photographed at $\times 200$ magnification. ${ }^{* *} \mathrm{P}<0.01$ and ${ }^{*} \mathrm{P}<0.05$ compared with control or sham group. (D-F), Plasma creatinine and KIM-1 concentrations. Plasma creatinine and KIM-1 concentrations were assessed in male C57/BL mice at different times after cisplatin administration (D), aristolochic acid administration (E), and UUO surgery (F) (n=5 per group). ${ }^{* *} \mathrm{P}<0.01$ and * $\mathrm{P}<0.05$ for the different kidney injury groups and the control group.

We examined KIM-1 and macrophage expression in kidney tissue samples from individuals with and without AKI and CKD. As shown in Fig. 6B, KIM-1 was not expressed in normal kidney. KIM-1-positive tubules surrounded by macrophages (CD68-positive) could be detected in AKI and CKD kidneys.

Kidney injury and fibrosis were associated with persistent proinflammatory macrophage expression

Accumulating experimental evidence indicates that macrophage phenotypes may drive kidney injury and fibrosis [17]. We examined changes in macrophage phenotypes further. In the three mouse models, the expression levels of proinflammatory macrophage 


\section{Cellular Physiology \\ Cell Physiol Biochem 2017;41:769-783 \\ \begin{tabular}{ll|l} 
and Biochemistry & Dublished on/ne: February 13, 2017 & $\begin{array}{l}\text { @ 2017 The Author(s). Published by S. Karger AG, Basel } \\
\text { www.karger.com/cpb }\end{array}$ \\
\hline
\end{tabular}}

Tian et al.: KIM-1 Regulates Macrophage in Nephropathy

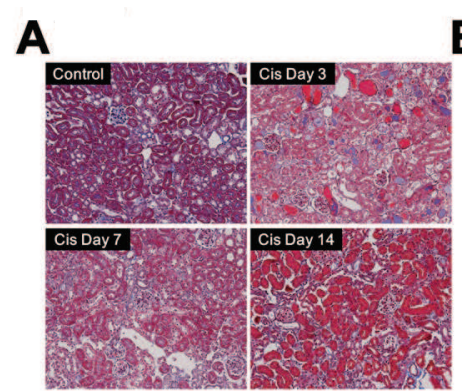

B

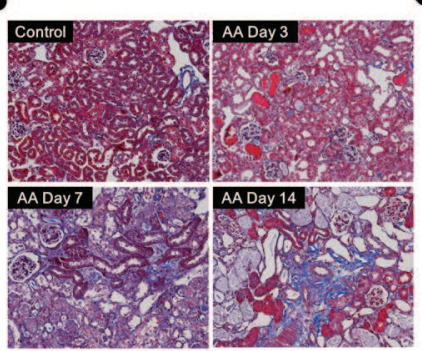

C
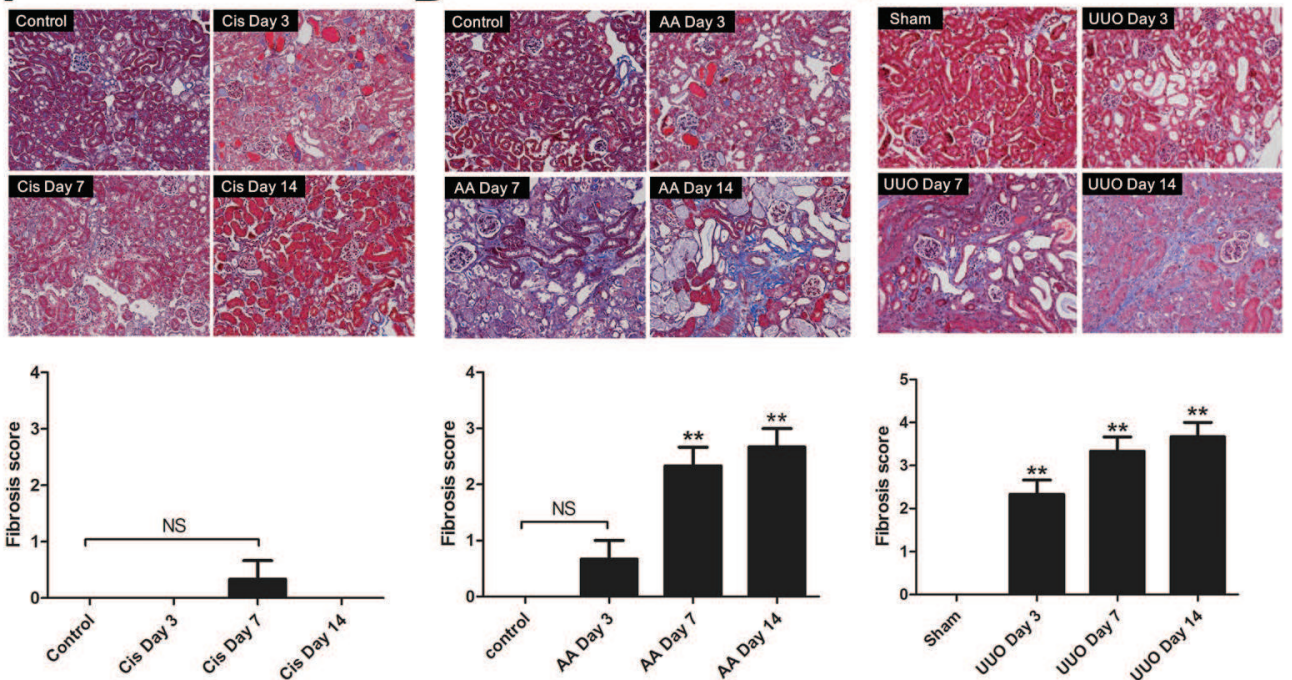

D

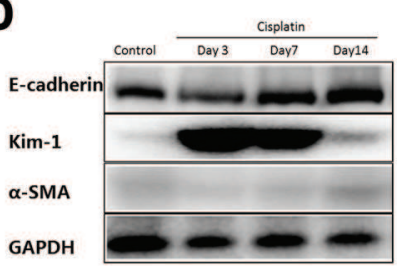

E

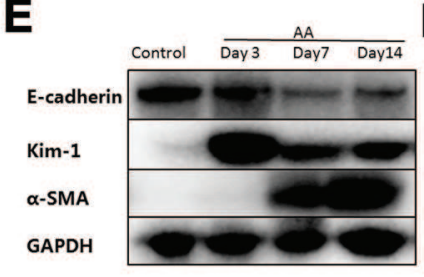

$\mathbf{F}$

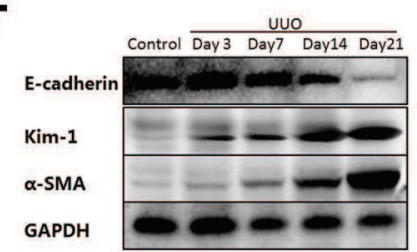

Fig. 4. Sustained KIM-1 expression was observed in renal fibrosis. (A-C), Masson trichrome staining of the kidney sections and quantification of renal fibrosis in vivo. The kidney sections were stained with Masson trichrome and photographed at $\times 200$ magnification after cisplatin administration (A), AA treatment (B), and UUO surgery (C). Western blot analysis of the kidney lysates from male C57/BL mice treated with cisplatin (D), aristolochic acid (E), and UUO surgery (F). ${ }^{* *} \mathrm{P}<0.01$ and $* \mathrm{P}<0.05$ for the different kidney injury groups and the control group.

cytokines in blood, such as TNF- $\alpha$ and IL-6, all of which are known to be released by activated proinflammatory macrophages, were increased at day 3 after kidney injury (Fig. 7,A-B). This finding was consistent with those regarding the increases in the mRNA expression levels of M1-dependent genes in renal cortex, such as IFN- $\gamma$ and iNOS (Fig. 7,E-F). In contrast, the expression levels of M2-dependent cytokines (IL-4 and IL-10) and genes (Arg-1 and MR) were decreased (Fig. 7,C-D,G-H). The trends in cytokine and macrophage gene expression reverted to their previous patterns at day 14 after cisplatin treatment. However, in the other two mouse models, M1 macrophage expression still predominated over M2 macrophage expression at day 14 after treatment.

\section{Involvement of MAPK signalling in KIM-1-induced macrophage migration and phenotype} transformation in vitro

Previous studies have shown that the MAPK signalling pathway is involved in macrophage activation [18]. To determine whether KIM-1 activates the MAPK signalling pathway, MAPK phosphorylation was analysed by western blotting. The results showed that treating Raw264.7 cells with recombinant KIM-1 significantly stimulated ERK1/2, p38, and JNK phosphorylation in these cells (Fig. 8,A). As shown in Fig. 8,B, KIM-1 treatment increased Raw264.7 cell migration compared with untreated control cells in transwell migration assay. When the cells were pretreated with specific MPAK inhibitors (U0126, SB203580, or SP600125), cell migration was significantly inhibited.

\section{KARGER}




\section{Cellular Physiology Cell Physiol Biochem 2017;41:769-783 \begin{tabular}{l|l|l|l|l}
\hline DOI: 10.1159/000458737 & (O) 2017 The Author(s). Published by S. Karger AG, Basel
\end{tabular}}

Tian et al.: KIM-1 Regulates Macrophage in Nephropathy

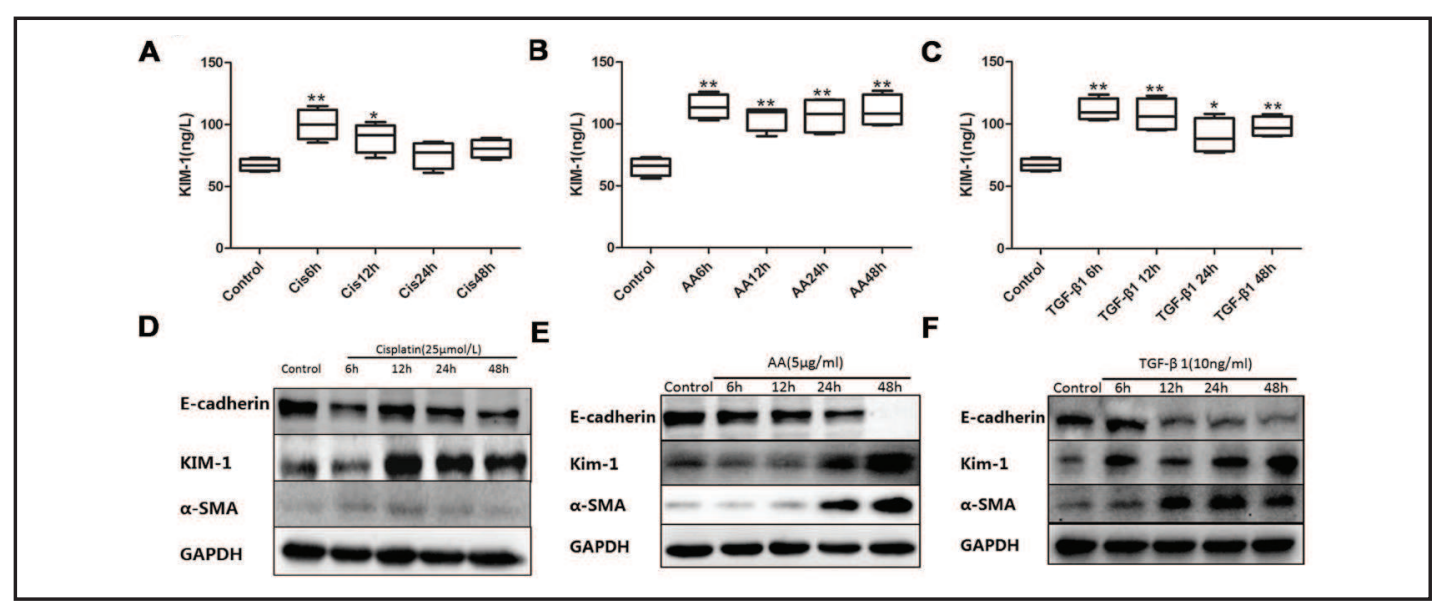

Fig. 5. KIM-1 expression and renal fibrosis in vitro. HK-2 cells were treated with cisplatin (25 $\mu$ mol/L), aristolochic acid (AA) $(5 \mu \mathrm{g} / \mathrm{ml})$, or TGF- $\beta 1$ (10 ng/ml). The HK-2 cell supernatants were assayed for KIM-1 expression after cisplatin administration (A), aristolochic acid administration (B), and TGF- $\beta 1$ administration (C) using an ELISA kit. Western blotting determined KIM-1 expression levels in the lysates from the HK-2 cells treated with cisplatin (D), AA (E), and TGF- $\beta 1(F) .{ }^{* *} \mathrm{P}<0.01$ and ${ }^{*} \mathrm{P}<0.05$ for the different injury groups and the control group.

Fig. 6. KIM-1-positive tubules were surrounded by Macrophages. (A) KIM-1 was costained with the macrophage marker F4/80 for immunofluorescence in mice treated with cisplatin, aristolochic acid, and UUO surgery. The kidney sections from these mice were photographed at $\times 200$ magnification and stained for KIM-1 (red) and the macrophage marker F4/80 (green). (B) KIM-1 was costained with the macrophage marker CD68 for immunofluorescence in human kidney samples. The kidney sections from humans were photographed at $\times 400$ magnification and stained for KIM-1 (green) and the macrophage marker CD68 (red). The normal kidney sections were obtained from paraneoplastic normal renal tissues.

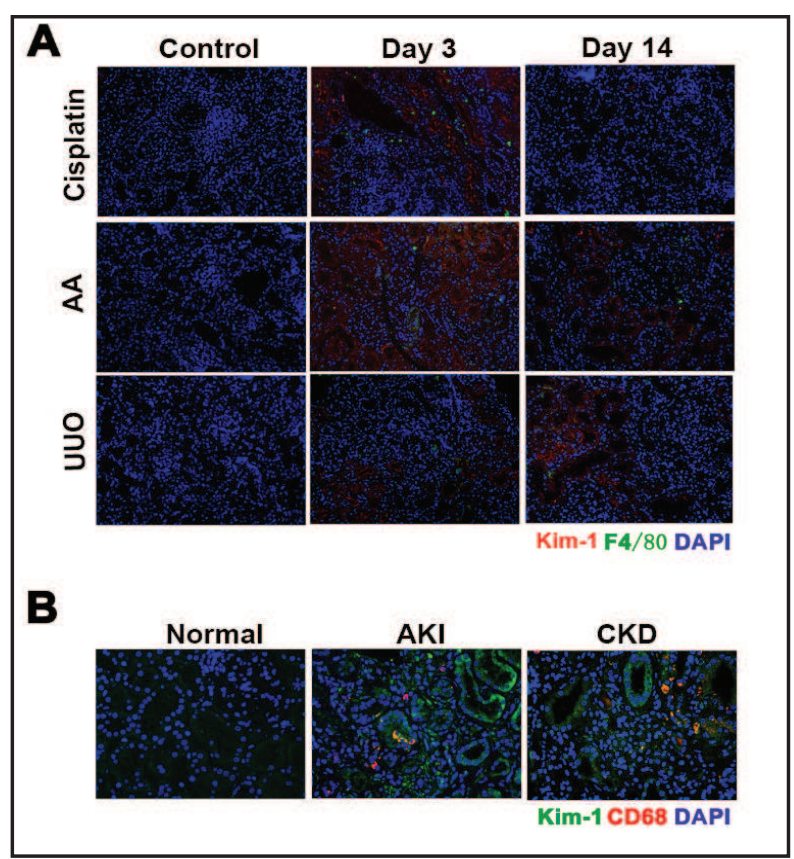

After exposure to recombinant KIM-1 for 12 hours, the mRNA expression levels of M1 markers, such as IFN- $\gamma$ and iNOS, were increased (Fig. 8,C-D). In contrast, the expression levels of M2 markers, such as Arg 1 and MR, were decreased (Fig. 8,E-F). KIM-1 seemed to activate macrophages towards the M1 phenotype. However, when the cells were pretreated with specific MAPK inhibitors (U0126, SB203580, or SP600125), the macrophage phenotypes reversed (Fig. 8,C-D,E-F).

\section{Discussion}

KIM-1 is widely recognized as an early biomarker of AKI [5]. Due to its potential utility as a therapeutic target and biomarker for kidney injury, KIM-1 has received increasing attention. Previous studies focused mostly on the diagnostic value of KIM-1, particularly the diagnostic 


\section{Cellular Physiology \\ Cell Physiol Biochem 2017;41:769-783 \begin{tabular}{ll|l} 
and Biochemistry & Dublisned on/ne: February 13, 2017 & $\begin{array}{l}\text { @ } 2017 \text { The Author(s). Published by S. Karger AG, Basel } \\
\text { www.karger.com/cpb }\end{array}$ \\
\hline
\end{tabular} \\ Tian et al.: KIM-1 Regulates Macrophage in Nephropathy}

A

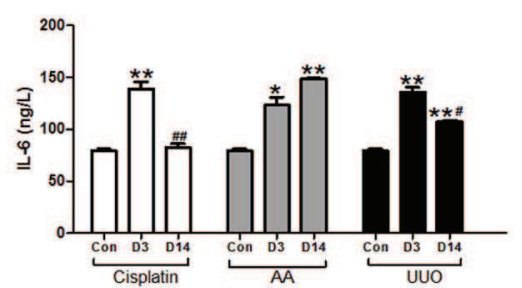

c

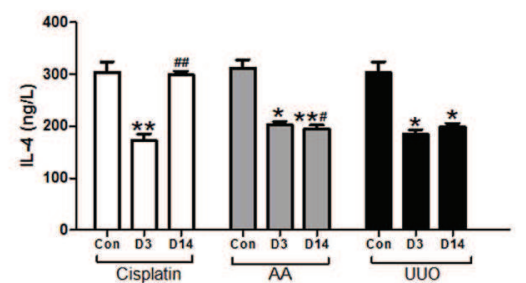

E

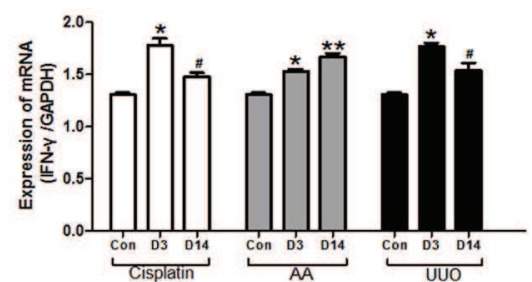

G

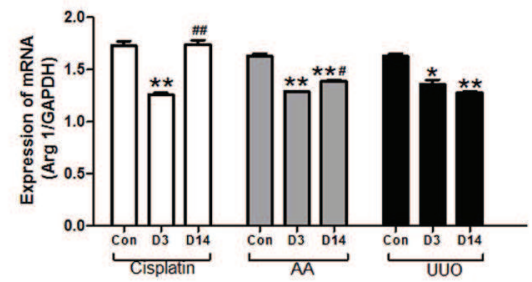

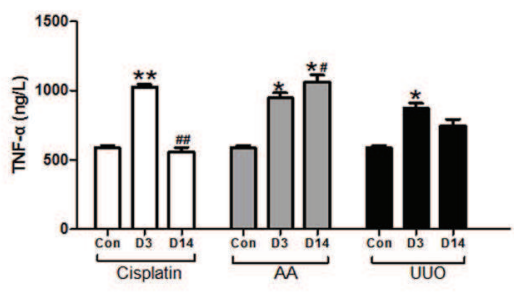

D
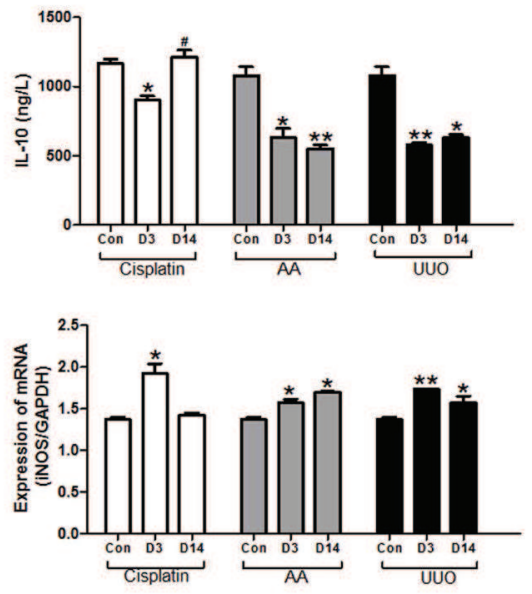

H

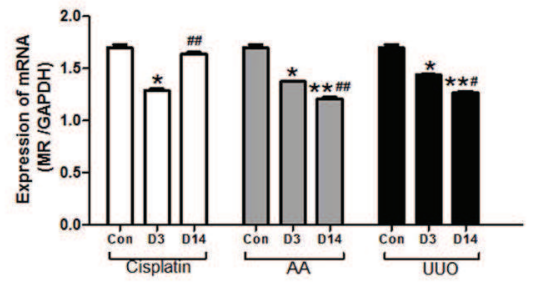

Fig. 7. Macrophage phenotype transition during renal injury and fibrosis. The blood levels of the proinflammatory cytokines (TNF- $\alpha$ and IL-6) released by M1 macrophages and the anti-inflammatory cytokines (IL-4 and IL-10) released by M2 macrophages in the three mouse models were quantified by ELISA kits (A-D). The renal mRNA expression levels of the indicated M1 markers (INF- $\gamma$ and iNOS) and M2 markers (Arg-1 and $\mathrm{MR})$ were detected by real-time RT-PCR $(\mathrm{E}-\mathrm{H}){ }^{* *} \mathrm{P}<0.01$ versus control group; ${ }^{*} \mathrm{P}<0.05$ versus control group; \#\#P<0.01 versus d3; \#P<0.05 versus d3. Abbreviations: TNF- $\alpha$, tumour necrosis factor- $\alpha$; IL-6, interleukin-6; IL-4, interleukin-4; IL-10, interleukin-10; IFN- $\gamma$, interferon- $\gamma$; iNOS, inducible nitric oxide synthase; Arg-1, arginase 1; MR, mannose receptor.

value of urinary KIM-1, in AKI. A few studies detected KIM-1 expression and suggested that it may be related to individual chronic kidney diseases, such as IgA nephropathy, lupus nephritis, or limited cases of type-1 diabetes $[19,20,16]$. Our cross-sectional study recruited patients with kidney diseases of various aetiologies, indicating that both plasma and urinary KIM-1 levels were significantly elevated in both AKI and CKD patients, thereby revealing that KIM-1 expression was also closely associated with chronic kidney injury. Moreover, we noted the existence of a significantly negative correlation between plasma KIM-1 expression and renal function in our study. These findings indicate that plasma KIM-1 may have clinical value in kidney injury. But the correlation was small and had no linearity. KIM-1 might be expressed after an insult as a very early reaction and the expression would be decreased soon afterwards even the damage still existed. In addition, the eGFR was not correlated with plasma KIM-1 or urinary KIM-1 expression in subset analysis, perhaps because of the small numbers of samples included in the subgroups.

Numerous studies have focused on the clinical value of KIM-1 at a particular time [6-8, 21-23]. Few studies have focused on the changes in KIM-1 expression that manifest during 


\section{Cellular Physiology Cell Physiol Biochem 2017;41:769-783 \begin{tabular}{l|l|l|l} 
DOI: 10.1159/000458737 & ( 2017 The Author(s). Published by S. Karger AG, Basel
\end{tabular}}

Tian et al.: KIM-1 Regulates Macrophage in Nephropathy

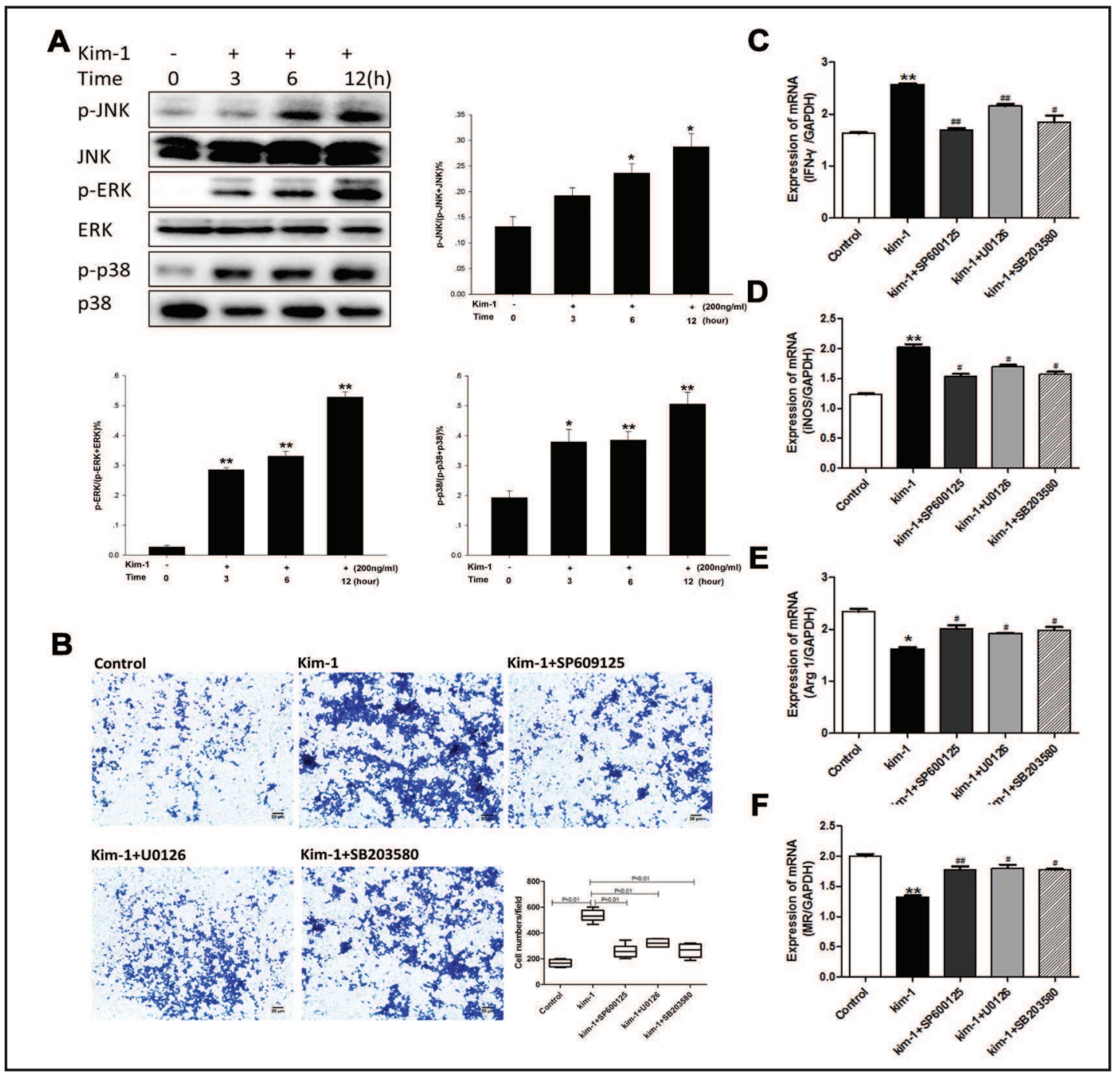

Fig. 8. Effects of KIM-1 expression on macrophage migration and phenotype transition. (A) Raw264.7 cells were incubated with recombinant KIM-1 (200 ng/ml). Phosphorylated phosphor-JNK, phosphor-ERK, and phosphor-p38 levels were observed by western blotting. JNK, ERK, and p38 expression levels were used as controls. (B) Macrophage migration assay was conducted using a 24-well transwell unit. The cells were loaded into the top chamber and pretreated with or without specific MAPK inhibitors (U0126 $10 \mu \mathrm{mol} / \mathrm{L}$, SB203580 $20 \mu \mathrm{mol} / \mathrm{L}$, or SP600125 $20 \mu \mathrm{mol} / \mathrm{L}$ ). After KIM-1 stimulation, the chambers were incubated for 12 hours. The cells that had migrated to the lower surfaces of the filter were fixed with $20 \%$ methanol for 30 min and stained with $0.2 \%$ crystal violet for $30 \mathrm{~min}$. The macrophages that had migrated were quantified by counting 5 random spots in each independent sample. (C-F) Characterization of the macrophages by mRNA expression showed increased mRNA levels of M1, but not M2, markers in Raw264.7 cells treated with KIM-1 after 12 hours. However, MAPK inhibitors (U0126, SB203580, or SP600125) could reverse the macrophage phenotype transitions. ${ }^{* *} \mathrm{P}<0.01$ versus control group; ${ }^{*} \mathrm{P}<0.05$ versus control group; $\# \# \mathrm{P}<0.01$ versus KIM1 group; \#P<0.05 versus KIM-1 group.

the occurrence and development of kidney injury [24, 25]. In this study, we established three different mouse models, including a cisplatin-induced AKI model, an aristolochic acid-induced renal fibrosis after AKI model, and a UUO-induced renal fibrosis model. These three mouse models represented different kidney injury and repair processes. However, the changes in KIM-1 expression that occurred in the kidney tissues were different from those that occurred in the plasma samples, indicating that the mechanism underlying KIM-1 expression 


\section{Cellular Physiology Cell Physiol Biochem 2017;41:769-783

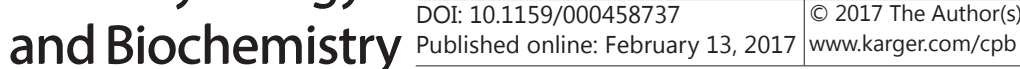 \\ Tian et al.: KIM-1 Regulates Macrophage in Nephropathy}

in kidney disease is intricate. KIM-1 can be detected in blood, a phenomenon that has been attributed to the loss of tubular cell polarity, increases in transepithelial permeability, and the disruption of actin cytoskeletal architecture in renal microvascular cells [16]. Kidney structural destruction varied in the three animal models; thus, blood KIM-1 expression levels varied across the different animal models. We used cisplatin to establish a model of acute kidney injury that could be healed. However, the mice that were treated with AA developed AKI at an early stage and progressed to renal fibrosis. Tissue KIM-1 expression in levels were elevated during the early stage of AKI in these two animal models, but the outcomes of the models were completely different. Sustained KIM-1 expression may be associated with kidney injury outcomes. In the UUO model, a classical renal fibrosis model, sustained KIM-1 expression was observed in renal fibrosis. The KIM-1 expression trends that were observed in kidney tissues in vivo suggested that sustained KIM-1 expression, not sustained increases in KIM-1 expression, was related to renal fibrosis. Taken together, these results indicated that KIM-1 may be involved in the occurrence and progression of kidney injury.

The findings of many studies indicate that KIM-1 is a sensitive and specific marker of kidney injury, as well as a predictor of prognosis $[6-8,16]$. In acutely injured kidneys, KIM1 is thought to confer a phagocytic phenotype on kidney epithelial cells that contributes to recovery by removing the debris of cells that died during the injury [26]. The results of our previous meta-analysis indicated that urinary KIM-1 was an efficient novel urinary biomarker for the diagnosis of AKI [8]. Ismail et al. [27] reported that KIM-1 provided endogenous protection against renal ischaemia-reperfusion injury by inhibiting G protein $\alpha 12$. However, these studies focused mainly on the expression and function of KIM-1 at certain disease stages. Our study observed the changes in KIM-1 expression during different stages of renal injury by performing clinical research and animal experiments. In the current study, we not only observed that KIM-1 expression increased during the early stage of kidney injury but also noted that KIM-1 expression was persistently elevated in renal fibrosis.

Our immunofluorescence staining results indicated that KIM-1-positive tubules were surrounded by macrophage infiltrates in damaged and fibrotic kidney regions. Furthermore, our in vitro study results indicated that KIM-1 expression was related to macrophage migration and was inclined to activate macrophages towards the M1 phenotype and that the MAPK signalling pathway was involved in the effects of KIM-1 on macrophages. Although the findings of cell experiments provided evidence that KIM-1 could promote macrophage migration and phenotype changes, our results could not show a clear link between KIM1 and macrophage phenotype transformation in vivo. These findings indicated that KIM-1 seemed to regulate macrophages via the MAPK signalling pathway in kidney disease.

Elevated KIM-1 expression has also been shown to occur near inflammatory cells and fibrotic tissue in CKD patients [11]. A recent study led by Benjamin Humphreys and Joseph Bonventre first demonstrated that chronic epithelial kidney injury molecule- 1 expression could cause murine kidney fibrosis [28]. This study provided strong evidence that KIM1 was related to CKD. A clinical study [16] about type-I diabetes found that blood KIM-1 expression was a biomarker for chronic kidney injury, a finding consistent with those of our cross-sectional study. On the basis of the results our study of three different mice models, we surmise that persistently high KIM-1 expression may be involved in renal fibrosis.

Macrophages are known to contribute to inflammation and fibrosis in many kidney diseases $[9,10,29,30]$. Based on our immunofluorescence staining results for our human kidney slides and mouse kidney injury slides, we determined that KIM-1-positive tubules were surrounded by macrophage infiltrates in damaged and fibrotic kidney regions. Previous studies have reported that KIM-1 and macrophages exhibit similar localization in kidney injury $[11,19]$. Our experiments regarding macrophages further demonstrated that KIM-1 could promote macrophage migration and phenotype changes.

Inflammation is a natural host defensive process that is largely regulated by macrophages during the innate immune response. MAPKs are major players in inflammatory responses, especially in macrophages [31]. A study focusing on chronic obstructive pulmonary disease reported that the p38 MAPK and ERK pathways mediated macrophage activation [32]. 


\section{Cellular Physiology Cell Physiol Biochem 2017;41:769-783

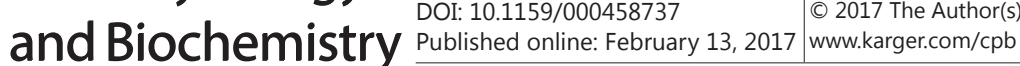 \\ Tian et al.: KIM-1 Regulates Macrophage in Nephropathy}

Previous studies have demonstrated that the MAPK signalling pathway was involved in regulating the inflammatory macrophage response towards lipopolysaccharide [33]. In vitro, we discovered that MAPK signalling pathways were involved in the effects of KIM-1 on macrophages. Therefore, we surmised that MAPK signalling pathways may play a primary role in KIM-1-mediated macrophage migration and phenotype transitions.

In this study, we focused mainly on the role of KIM-1 in kidney disease. KIM-1 is an early biomarker of kidney injury whose function in the early stage of kidney injury is still unclear. However, a recent study reported that KIM-1 exerts anti-inflammatory effects by downregulating innate immunity and inflammation in the early stage of kidney injury [13]. KIM-1 may have various biological functions. Additional investigations should be conducted for fully understand the role of KIM-1 in renal injury.

Taken together, our findings indicated that KIM-1 is a valuable biomarker for both acute and chronic kidney injury. KIM-1 played an important role in macrophage migration and phenotype transition, and the MAPK signalling pathway may be involved in this process.

\section{Acknowledgements}

The study was sponsored by Hong Kong, Macao, and Taiwan S\&T Cooperation Program of China (2014DFT30090), the National Natural Science Foundation of China (81373865 and 81573748 ), as well as by grants (16XD1401900, 12401906400, 13401906100 and 14140903200) from the Science and Technology Commission of Shanghai Municipality, China. Program ZYSNXD012-RC-ZXY017 and 20154Y0003 from the Shanghai Health Bureau and 15020110599 from Chinese medical association special foundation for medical science were also included.

\section{Disclosure Statement}

None.

\section{References}

1 Zhang L, Wang F, Wang L, Wang W, Liu B, Liu J, Chen M, He Q, Liao Y, Yu X, Chen N, Zhang JE, Hu Z, Liu F, Hong D, Ma L, Liu H, Zhou X, Chen J, Pan L, Chen W, Wang W, Li X, Wang H: Prevalence of chronic kidney disease in China: a cross-sectional survey. Lancet 2012;379:815-822 .

2 Klarenbach SW, Tonelli M, Chui B, Manns BJ: Economic evaluation of dialysis therapies. Nat Rev Nephrol 2014;10:644-652.

3 Berger K, Moeller M J: Mechanisms of Epithelial Repair and Regeneration After Acute Kidney Injury. Semin Nephrol 2014;34:394-403.

-4 Ichimura T, Bonventre JV, Bailly V, Wei H, Hession CA, Cate RL, Sanicola M: Kidney injury molecule1(KIM-1), a putative epithelial cell adhesion molecule containing a novel immunoglobulin domain, is upregulated in renal cells after injury. J Biol Chem 1998;273:4135-4142.

-5 Bonventre JV, Yang L: Kidney injury molecule-1. Curr Opin Crit Care 2010;16:556-561.

-6 Xie Y, Xue W, Shao X, Che X, Xu W, Ni Z, Mou S: Analysis of a Urinary Biomarker Panel for Obstructive Nephropathy and Clinical Outcomes. PLoS One 2014;9:e112865.

-7 Xue W, Xie Y, Wang Q, Xu W, Mou S, Ni Z: Diagnostic performance of urinary kidney injury molecule-1 and neutrophil gelatinase-associated lipocalin for acute kidney injury in an obstructive nephropathy patient. Nephrology (Carlton) 2014;19:186-194.

8 Shao X, Tian L, Xu W, Zhang Z, Wang C, Qi C, Ni Z, Mou S: Diagnositic value of urinary kidney injury molecule 1 for acute kidney injury: a meta-analysis. PLoS One 2014;9:e84131.

-9 Wang YP, Harris DC: Macrophages in Renal Disease. J Am Soc Nephrol 2011;22:21-27.

10 Sean Eardley K, Cockwell P: Macrophages and progressive tubulointerstitial disease. Kidney Int 2005;68:437-455.

-11 van Timmeren MM, van den Heuvel MC, Bailly V, Bakker SJ, van Goor H, Stegeman CA: Tubular kidney injury molecule-1 (KIM-1) in human renal disease. J Pathol 2007;212:209-217.

12 Lim AL, Tang SC, Lai KN, Leung JC: Kidney injury molecule-1: more than just an injury marker of tubular epithelial cells? J Cell Physiol 2013;228:917-924. 


\section{Cellular Physiology Cell Physiol Biochem 2017;41:769-783

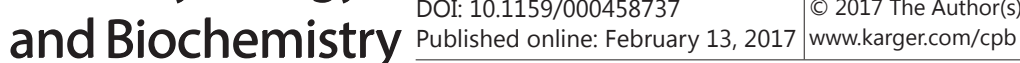

Tian et al.: KIM-1 Regulates Macrophage in Nephropathy

13 Yang L, Brooks CR, Xiao S, Sabbisetti V, Yeung MY, Hsiao LL, Ichimura T, Kuchroo V, Bonventre JV: KIM-1mediated phagocytosis reduces acute injury to the kidney. J Clin Invest 2015;125:1620-1636.

14 Xu W, Shao X, Tian L, Gu L, Zhang M, Wang Q, Wu B, Wang L, Yao J, Xu X, Mou S, Ni Z: Astragaloside IV Ameliorates Renal Fibrosis via the Inhibition of Mitogen-Activated Protein Kinases and Antiapoptosis In Vivo and In Vitro. J Pharmacol Exp Ther 2014;350:552-562.

15 Levey AS, Coresh J, Greene T, Marsh J, Stevens LA, Kusek JW, Van Lente F, Chronic Kidney Disease Epidemiology Collaboration: Expressing the Modification of Diet in Renal Disease Study Equation for Estimating Glomerular Filtration Rate with Standardized Serum Creatinine Values. Clin Chem 2007;53:766-772.

16 Sabbisetti VS, Waikar SS, Antoine DJ, Smiles A, Wang C, Ravisankar A, Ito K, Sharma S, Ramadesikan S, Lee M, Briskin R, De Jager PL, Ngo TT, Radlinski M, Dear JW, Park KB, Betensky R, Krolewski AS, Bonventre JV:Blood Kidney Injury Molecule-1 Is a Biomarker of Acute and Chronic Kidney Injury and Predicts Progression to ESRD in Type I Diabetes. J Am Soc Nephrol 2014;25:2177-2186.

-17 Susnik N, Sörensen-Zender I, Rong S, von Vietinghoff S, Lu X, Rubera I, Tauc M, Falk CS, Alexander WS, Melk A, Haller H, Schmitt R: Ablation of proximal tubular suppressor of cytokine signaling 3 enhances tubular cell cycling and modifies macrophage phenotype during acute kidney injury. Kidney Int 2014;85:13571368.

18 Yang H, Young DW, Gusovsky F, Chow JC: Cellular events mediated by lipopolysaccharide-stimulated tolllike receptor 4. MD-2 is required for activation of mitogen-activated protein kinases and Elk-1. J Biol Chem 2000;2759:20861-20866.

19 Lin Q Chen Y, Lv J, Zhang H, Tang J, Gunaratnam L, Li X, Yang L: Kidney injury molecule-1 expression in IgA nephropathy and its correlation with hypoxia and tubulointerstitial inflammation. Am J Physiol Renal Physiol 2014;306:885-895.

20 Nozaki Y, Kinoshita K, Yano T, Shiga T, Hino S, Niki K, Kishimoto K, Funauchi M, Matsumura I: Estimation of kidney injury molecule-1 (Kim-1) in patients with lupus nephritis. Lupus 2014:23:769-777.

-21 Vaidya VS, Ramirez V, Ichimura T, Bobadilla NA, Bonventre JV: Urinary kidney injury molecule-1: a sensitive quantitative biomarker for early detection of kidney tubular injury. Am J Physiol Renal Physiol 2006;290:517-529.

22 Han WK, Bailly V, Abichandan R, Thadhan R, Bonventre JV: Kidney injurymolecule-1(KIM-1): a novel biomarker for human renal proximal tubule injury. Kidney Int 2002;62:237-244.

-23 Vaidya VS, Ferguson MA, Bonventre JV: Biomarkers of acute kidney injury. Annu Rev Pharmacol Toxicol 2008;48:463-493.

24 Kusaba T, Lalli M, Kramann R, Kobayashi A, Humphreys BD: Differentiated kidney epithelial cells repair injured proximal tubule. Proc Natl Acad Sci USA 2014;111:1527-1532.

25 Bonventre JV: Can we target tubular damage to prevent renal function decline in diabetes? Semin Nephrol 2012;32:452-462.

-26 Ichimura T, Asseldonk EJ, Humphreys BD, Gunaratnam L, Duffield JS, Bonventre JV:Kidney injury molecule- 1 is a phosphatidylserine receptor that confers a phagocytic phenotype on epithelial cells. J Clin Invest 2008;118:1657-1668.

27 Ismail OZ, Zhang X, Wei J, Haig A, Denker BM, Suri RS, Sener A, Gunaratnam L:Kidney Injury Molecule-1 Protects against Ga12 Activation and Tissue Damage in Renal Ischemia-Reperfusion Injury. Am J Pathol 2015;185:1207-1215.

28 Humphreys BD, Xu F, Sabbisetti V, Grgic I, Movahedi Naini S, Wang N, Chen G, Xiao S, Patel D, Henderson JM, Ichimura T, Mou S, Soeung S, McMahon AP, Kuchroo VK, Bonventre JV:Chronic epithelial kidney injury molecule-1 expression causes murine kidney fibrosis. J Clin Invest 2013;123:4023-4035.

-29 Silva RC, Landgraf MA, Corrêa-Costa M, Semedo P, Cenedeze MA, Pacheco-Silva A, Landgraf RG, Câmara NO: Acute Kidney injury reduces phagocytic and microbicidal capacilities of alveolar macrophages. Cell Physiol Biochem 2013;31:179-188.

-30 Pan B, Liu G, Jiang Z, Zheng D. Regulation of renal fibrosis by macrophage polarization. Cell Physiol Biochem 2015;35:1062-1069.

- 31 Yang Y, Kim SC, Yu T, Yi YS, Rhee MH, Sung GH, Yoo BC, Cho JY: Functional Roles of p38 Mitogen-Activated Protein Kinase in Macrophage-Mediated Inflammatory Responses. Mediators Inflamm 2014;2014:352327.

- 32 Gu W, Song L, Li XM, Wang D, Guo XJ, Xu WG: Mesenchymal stem cells alleviate airway inflammation and emphysema in COPD through down-regulation of cyclooxygenase-2 via p38 and ERK MAPK pathways. Sci Rep 2015;5:8733.

-33 Bode JG, Ehlting C, Haussinger D: The macrophage response towards LPS and its control through the p38MAPK-STAT3 axis. Cell Signal 2012;24:1185-1194. 